\title{
Étude des compléments différés à l'oral à l'interface syntaxe - prosodie
}

\author{
Noalig Tanguy \\ Université Paris 3 Sorbonne Nouvelle - Laboratoire LaTTiCe UMR 8094 \\ noaligtanguy@gmail.com
}

\section{Introduction}

De nombreux travaux sur des larges corpus de français parlé ont vu le jour ces dernières années et ont permis de mettre en lumière des structures bien particulières, trop souvent mal définies, comme par exemple le phénomène de l'ajout, constituant une difficulté à une description en termes de phrase et illustré par l'exemple (1), dans lequel les doubles barres obliques indiquent un rupture prosodique :

(1) je sais pas comment ils font // les prévisions [oral]

Tout analyste travaillant avec une unité telle que la phrase comme outil de description et de segmentation rencontre très vite le problème de la délimitation des unités phrastiques dans un texte. Même si nous défendons l'idée, à l'instar de Le Goffic (2001), que la phrase ne se définit pas par ses contours mais par son centre, nous reconnaissons que le niveau prosodique peut interférer sur les découpages syntaxiques.

Le phénomène de l'ajout est depuis longtemps étudié à travers des exemples de la langue écrite, tout d'abord sous le terme d'épexégèse (Bally, 1944), au sens d'explication ajoutée appelée à compléter, préciser, expliquer ou désambiguïser une première construction énoncée, ou encore sous l'appellation « hyperbate » (Bonhomme, 1998), comme figure de style réalisée en fin de phrase et ajoutant un élément à un ensemble en apparence complet syntaxiquement et sémantiquement. Ce phénomène a fait plus récemment l'objet de nombreux travaux pour le système oral (Sabio, 2006 ; Deulofeu, 2009 ; BlancheBenveniste, 1993, 2002, 2003 ; etc.).

Tout travail de structuration phrastique de textes oraux soulève immédiatement la difficulté d'interprétation syntaxique des constituants périphériques tels que le groupe prépositionnel "pour en faire un quartier tranquille » en (2), susceptible de connaître différentes lectures : (i) élément intégré à la structure qui précède, (ii) élément détaché ou (iii) élément autonome.

(2) ils ont essayé de casser le passage dans le quartier pour en faire un quartier tranquille [CFPP2000: Nicole Noroy $\left.14^{\mathrm{e}}\right]^{1}$

Nous emploierons pour notre description l'expression de «compléments différés » empruntée à BlancheBenveniste (1997 : 114) pour tout segment en marge des complémentations canoniques et marquant une rupture de linéarité dans le discours en cours de formulation.

Un complément peut être différé par des moyens divers : par la prosodie d'une part (3), et/ou par la présence d'un marqueur discursif tel que «mais », « enfin », « surtout », « d'ailleurs », etc., d'autre part (4).

(3) il me reste un morceau à mettre là // pour fermer euh le tour du cadre // [GRE03 : doreur]

(4) ça m'avait frappée mais mais comme un coup de tonnerre [CTFP : 35]

Partant de l'idée qu'il faut rendre compte à la fois, et des relations de type rectionnel ou autre existant entre les éléments, et des effets produits par le locuteur, intonatifs ou graphiques, nous souhaitons, à travers cet article, étudier le fonctionnement des compléments différés à l'oral, dans une approche couplant les niveaux syntaxique et prosodique. 


\section{Segmentation syntaxique vs segmentation prosodique}

Afin de déterminer le cadre dans lequel nous nous situons, nous commencerons par présenter les critères de segmentation retenus pour nos découpages syntaxiques et prosodiques.

\subsection{La phrase comme unité de segmentation syntaxique}

Notre modèle d'analyse syntaxique s'appuie sur les notions de grammaire et de phrase. Nous définissons cette dernière comme une prédication en acte, assortie d'une modalité d'énonciation (Le Goffic, 1993 \& 2008), c'est-à-dire qu'une phrase s'articule autour d'un prédicat, actualisé dans le discours par une modalité énonciative (assertive, interrogative ou injonctive). La phrase est donc une unité à la fois énonciative et syntaxique. Nous entendons par prédication l'opération sémantico-syntaxique de construction d'un énoncé, la mise en relation d'un sujet et d'un prédicat. Nous distinguons cinq manière de prédiquer (Tanguy, 2009) : une prédication d'existence, une prédication d'attribution, une prédication de localisation, une prédication d'activité et une prédication de cognitivité. Le rôle de prédicat peut être tenu par un groupe verbal ou par un constituant averbal. La phrase réalise ainsi un prédicat, lié ou non à un sujet de manière explicite, $\mathrm{au}(\mathrm{x})$ quel(s) se rattachent des éléments périphériques, avant ou après, plus ou moins extérieurs au prédicat. Une phrase véhicule donc une prédication et tout ce qui se rattache à cette prédication, et ne se limite pas à la sphère rectionnelle autour du verbe.

Pour notre modèle, nous reprendrons les termes introduits et employés par Blanche-Benveniste (1997 \& 2002 ; et al., 1990), à savoir les éléments « noyau », " préfixe », « suffixe » et « infixe », appliqués à un modèle syntaxique autour de la notion de phrase. Nous ne retiendrons ainsi du modèle aixois que la terminologie et non les contenus. De la sorte, une phrase comporte nécessaiement un centre prédicatif (le noyau) éventuellement, mais pas nécessairement, encadré d'éléments idéalement extra-prédicatifs (les affixes). Le noyau réalise le prédicat et très souvent son sujet en structure liée. Il comprend également les circonstants internes au prédicat (dits «intra-prédicatifs »). Les affixes, selon leur place, peuvent être préfixes, suffixes ou infixes.

\subsection{La période comme unité de segmentation prosodique}

$\mathrm{Au}$ niveau prosodique, nous travaillerons à partir d'unités d'intégration prosodique supérieures : la période intonative telle que la définissent Lacheret-Dujour et Victorri (2002 ; Lacheret-Dujour, 2003 ; Avanzi \& Lacheret-Dujour, à par. ; Avanzi, 2005 \& 2008).

A ce niveau, différents points sont expertisés : (i) la durée de la pause et plus précisément de l'intervalle entre deux portions de F0, (ii) l'amplitude du "geste », c'est-à-dire la différence de hauteur entre le dernier extremum de F0 et la moyenne de F0 sur toute la portion de signal qui précède la pause, (iii) l'amplitude du « saut », c'est-à-dire la différence de hauteur entre la dernière valeur (dernier extremum) de F0 précédant la pause et la première valeur de F0 suivant la pause, (iv) la présence d'un allongement lié à une hésitation (d'un « euh ») juste avant ou juste après la pause, ce qu'illustre la figure suivante : 

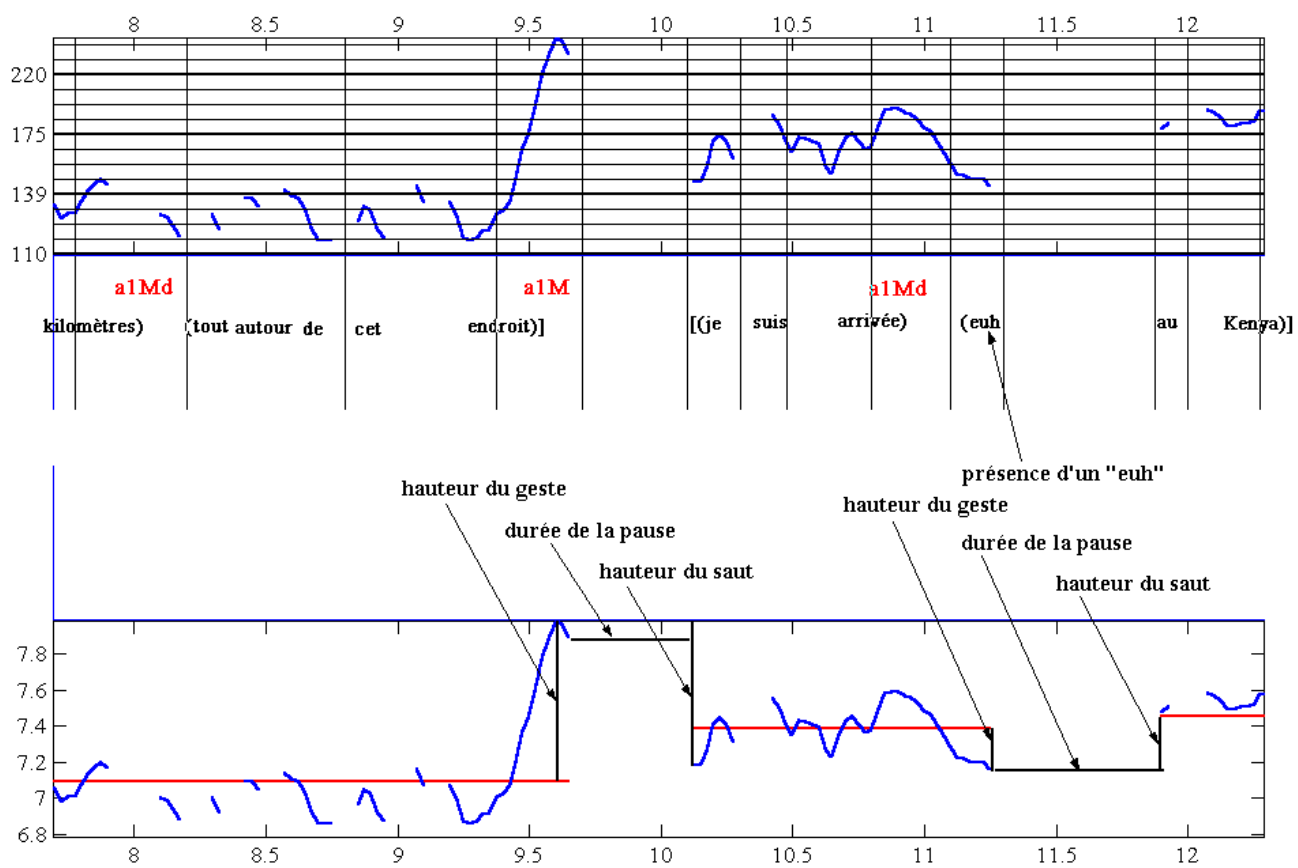

Figure $1:$ Illustration des quatre paramètres utilisés pour le découpage automatique en périodes intonatives, d'après Lacheret-Dujour \& Victorri (2002 : 63). Sortie Logiciel Analor (Avanzi et al., 2008).

Il y a ainsi segmentation en période si quatre conditions sont réunies : (i) la durée de la pause dépasse un seuil de l'ordre de $300 \mathrm{~ms}$, (ii) l'amplitude du «geste» dépasse un seuil de l'ordre de $4 \mathrm{dt}$, (iii) l'amplitude du «saut» dépasse un seuil de l'ordre de $3 \mathrm{dt}$, (iv) il n'y a pas de «euh » à la proximité immédiate de la pause.

\subsection{L'apport de la prosodie à la syntaxe}

L'analyse prosodique permet d'isoler des consituants averbaux que l'approche syntaxique, sans confrontation avec l'enregistrement du texte, n'avait pas repérés comme autonomes :

(5) d'ailleurs c'est à peu près le la configuration qu'ils ont gardée pour créer il y a quelques années ce fameux quartier vert // qui finalement a fini par tuer le quartier // parce qu'ils ont coupé totalement la la circulation // [CFPP2000 : Nicole Noroy 14 ${ }^{\mathrm{e}}$ ]

La question est alors de savoir si l'autonomie suggérée par la prosodie peut l'être également au niveau syntaxique. Nous pensons en effet que la prise en compte de données prosodiques est indispensable pour l'analyse de certains constituants tels que ceux relevés en (5), oscillant entre trois pôles : (i) intégré, (ii) détaché ou (iii) autonome.

\section{Typologie catégorielle}

Le phénomène du détachement à droite concerne tout syntagme susceptible d'entretenir une relation plus ou moins dépendante avec son contexte de gauche, des éléments rectionnels aux circonstants. Les subordonnées relatives, au fonctionnement identique à celui des adjectifs (ou groupes adjectivaux), constituent une catégorie bien particulière d'ajouts en produisant un effet discursif tout à fait particulier. Nous distinguerons trois ensembles de compléments différés : (i) les complément différés nominaux, (ii) les compléments différés adjectivaux, (iii) les compléments différés adverbiaux. 


\subsection{Les compléments différés nominaux}

Les complément différés nominaux sont équivalents à un groupe nominal et exercent des fonctions nominales au niveau de la phrase (complément direct, etc.) et à un niveau inférieur (apposition, etc.).

Dans l'extrait (6) :

(6) je travaillais pour une usine - Coder la même qu'il y a à Marseille /ou, X/à Saint-Marcel mais - à la Garenne Bezons à Paris - [CTFP : 27]

les groupes nominaux finaux se situent entre prédication seconde et autonome (première).

(i) L'extrait peut être lu en une seule unité syntaxique. Il s'agit alors d'une phrase simple constituée autour de la structure « sujet - verbe - circonstant».

\begin{tabular}{|l|l|l|}
\hline Préfixe(s) & Noyau & Suffixe(s) \\
\hline & $\begin{array}{l}\text { je travaillais pour une usine - Coder la } \\
\text { même qu'ily à Marseille lou, X/ à Saint- } \\
\text { Marcel mais - à la Garenne Bezons à } \\
\text { Paris }\end{array}$ & \\
\hline
\end{tabular}

(ii) Une prédication seconde, comme constituant apposé détaché en fin de phrase, est analysée dans notre modèle comme un suffixe :

\begin{tabular}{|l|l|l|}
\hline Préfixe(s) & Noyau & Suffixe(s) \\
\hline & je travaillais pour une usine- & $\begin{array}{l}\text { Coder la même qu'il y a ̀̀ } \\
\text { Marseille /ou, X/ à Saint- } \\
\text { Marcel mais - à la } \\
\text { Garenne Bezons à Paris }\end{array}$ \\
\hline
\end{tabular}

(iii) Enfin, les deux derniers constituants peuvent correspondre à des prédicats substantivaux attributifs à sujet implicite (Lefeuvre, 1999). Le référent du sujet est alors présent dans la phrase précédente. Les prédicats renvoient tous deux à « une usine ».

\begin{tabular}{|l|l|l|}
\hline Préfixe(s) & Noyau & Suffixe(s) \\
\hline & je travaillais pour une usine - & \\
\hline & Coder & \\
\hline & $\begin{array}{l}\text { la même qu'il y a à Marseille /ou, X/ à } \\
\text { Saint-Marcel mais - à la Garenne Bezons } \\
\text { à Paris }\end{array}$ \\
\hline
\end{tabular}

Le caractère averbal permet dans ce cas de pointer sur l'élément le plus informatif. Ainsi, l'information se fait de plus en plus précise avec une progression de l'apport informatif en trois temps :

$$
\begin{aligned}
& 1 \rightarrow \text { Je travaillais pour une usine. } \\
& 2 \rightarrow \text { Cette usine est Coder. } \\
& 3 \rightarrow \text { Cette usine Coder est la même qu'il y a à Marseille... }
\end{aligned}
$$




\subsection{Les compléments différés adjectivaux}

Les compléments différés adjectivaux sont équivalents à des adjectifs ou à des groupes adjectivaux et exercent des fonctions adjectivales (épithètes, appositions, etc.).

En (7):

(7) c'était souvent ou des gens d'une cinquantaine d'années donc euh plus d'enfants à charge ayant leur appartement donc très peu regardants [CFPP2000 : Nicole Noroy $14^{\mathrm{e}}$ ]

nous observons différents emplois possibles pour les ensembles « ayant leur appartement donc » et « très peu regardants ». Nous insisterons uniquement ici sur le groupe adjectival « très peu regardants », en admettant que le groupe participial qui lui précède soit intonativement lié à la construction "c'était souvent ou des gens d'une cinquantaire d'années donc euh plus d'enfants à charge ». L'intonation nous indique que l'adverbe « donc » porte sur ce qui précède et non ce qui suit.

(i) Nous pouvons analyser l'ensemble «très peu regardants » comme un élément lié, groupe épithète se rapportant au syntagme nominal « des gens d'une cinquantaine d'années ». Dans ce cas, il appartient au noyau.

\begin{tabular}{|l|l|l|}
\hline Préfixe(s) & Noyau & Suffixe(s) \\
\hline & $\begin{array}{l}\text { c'était souvent ou des gens d'une } \\
\text { cinquantaine d'années donc euh plus } \\
\text { d'enfants à charge ayant leur appartement } \\
\text { donc très peu regardants }\end{array}$ & \\
\hline
\end{tabular}

(ii) Une lecture en deux phrases distinctes peut également être envisagée. Le groupe adjectival constitue dans ce cas un prédicat averbal attributif à sujet implicite (Lefeuvre, 1999) dont le référent renvoie au syntagme « des gens d'une cinquantaine d'années ».

\begin{tabular}{|l|l|l|}
\hline Préfixe(s) & Noyau Suffixe(s) \\
\hline & $\begin{array}{l}\text { c'était souvent ou des gens d'une } \\
\text { cinquantaine d'années donc euh plus } \\
\text { d'enfants à charge ayant leur appartement } \\
\text { donc }\end{array}$ & \\
\hline & très peu regardants & \\
\hline
\end{tabular}

Un jugement est émis. L'énoncé est asserté. Nous verrons plus loin dans notre démonstration qu'une seconde possibilité, et vraisembleblement plus juste, consiste à analyser ce nouveau segment comme elliptique, structuré à partir d'une construction prédicative $\varnothing$ correspondant à la réactulisation de la prédication précédente. La construction ne comporte pas d'opération "sujet + prédicat » mais reste tout de même interprétable comme prédication par recours au contexte, en l'occurrence ici précédent.

(iii) Entre ces deux possibilités, une troisième pose le groupe adjectival comme détaché, apposé, en position de suffixe, comme suffixe régi (Deulofeu, 2003).

\begin{tabular}{|l|l|l|}
\hline Préfixe(s) & Noyau & Suffixe(s) \\
\hline & $\begin{array}{l}\text { c'était souvent ou des gens d'une } \\
\text { cinquantaine d'années donc euh plus } \\
\text { d'enfants à charge ayant leur appartement } \\
\text { donc }\end{array}$ & \\
\hline
\end{tabular}


Nous avons ainsi un degré d'autonomie plus ou moins marqué pour le groupe adjectival «très peu regardants » qui connaît un emploi d'épithète, d'apposition ou de prédicat. L'interprétation au niveau de l'information est différente selon les emplois.

Dans l'emploi lié, le locuteur pose comme information qu'il y a des gens d'une cinquantaine d'années. Ces personnes sont immédiatement qualifiées de très peu regardantes. L'assertion pose l'existence de gens d'une cinquantaine d'années très peu regardants.

Dans l'emploi détaché, le locuteur pose dans un premier temps le fait qu'il y a des gens d'une cinquantaine d'années et dans un second temps il précise que ces personnes sont très peu regardantes. Cependant dans ce cas, l'énonciation du suffixe est préparée par l'intonation (contour continuatif sur l'élément qui précède). En énonçant la prédication " c'était souvent des gens d'une cinquantaine d'années », le locuteur prévoit de lui adjoindre une qualification, mais de manière détachée.

Dans un emploi autonome, l'effet est encore différent. Le locuteur pose toujours dans un premier temps le fait qu'il s'agit de gens d'une cinquantaine d'années. Cependant il ajoute après-coup une qualification et cette attribution n'est pas amorcée puisque la première prédication est énoncé avec un contour conclusif. Ce contour conclusif et la fin d'une période intonative marquent la clôture de la phrase qui précède pour le producteur comme pour le récepteur. L'ajout ouvre une nouvelle unité prosodique et syntaxique même si le complément différé poursuit la phrase qui vient d'être achevée. Puisque celle-ci est achevée, l'adjectif énoncé ensuite ouvre nécessairement une nouvelle phrase. Ce dernier emploi doit être lu au sens de :

c'était souvent des gens d'une cinquantaine d'années || très peu regardants [ces gens]

c'était souvent des gens d'une cinquantaine d'années || [ils étaient] très peu regardants

Les différentes possibilités peuvent être illustrées par la graphie :

C'était souvent des gens d'une cinquantaine d'années très peu regardants.

C'était souvent des gens d'une cinquantaine d'années, très peu regardants.

C'était souvent des gens d'une cinquantaine d'années. Très peu regardants.

\subsection{Les compléments différés adverbiaux}

Les compléments différés adverbiaux sont équivalents à un adverbe et exercent des fonctions adverbiales (circonstants, localisations essentielles, etc.).

Dans l'exemple (2) :

(2) ils ont essayé de casser le passage dans le quartier pour en faire un quartier tranquille [CFPP2000: Nicole Noroy $14^{\mathrm{e}}$ ]

le groupe prépositionnel «pour en faire un quartier tranquille» est susceptible de connaitre trois interprétations distinctes.

(i) S'il est intégré au noyau, la portée du circonstant est dite « intra-prédicative ».

\begin{tabular}{|l|l|l|}
\hline Préfixe(s) & Noyau & Suffixe(s) \\
\hline & $\begin{array}{l}\text { ils ont essayé de casser le passage dans le } \\
\text { quartier pour en faire un quartier } \\
\text { tranquille }\end{array}$ & \\
\hline
\end{tabular}


(ii) En position détachée et ainsi analysé comme un suffixe, le circonstant est dit « extra-prédicatif».

\begin{tabular}{|l|l|l|}
\hline Préfixe(s) & Noyau & Suffixe(s) \\
\hline & $\begin{array}{l}\text { ils ont essayé de casser le passage dans le } \\
\text { quartier }\end{array}$ & $\begin{array}{l}\text { pour en faire un quartier } \\
\text { tranquille }\end{array}$ \\
\hline
\end{tabular}

(iii) Analysé comme un élément autonome, le groupe prépositionnel constituerait dans ce cas une sorte de prédicat focalisé au sens de «c'est pour en faire un quartier tranquille qu'ils ont essayé de casser le passage dans le quartier » ou une construction elliptique calquée sur la phrase qui précède (cf. infra).

\begin{tabular}{|l|l|l|}
\hline Préfixe(s) & Noyau & Suffixe(s) \\
\hline & $\begin{array}{l}\text { ils ont essayé de casser le passage dans le } \\
\text { quartier }\end{array}$ & \\
\hline & pour en faire un quartier tranquille & \\
\hline
\end{tabular}

\title{
4 Aspects discursifs, pragmatiques \& informationnels
}

Un complément différé conduit bien souvent à une modification dans le domaine énonciatif, avec des effets polyphoniques (explication, réorientation discursive, commentaire à orientation négative, développement, précision, etc.) comme le souligne Combettes (2007 : 128) :

\begin{abstract}
« A s'en tenir à ce type de marqueurs, on peut en effet constater que la fragmentation due à la présence d'ajout correspond à une importance accrue du niveau énonciatif et du niveau informationnel, la phrase tendant à devenir un palier de traitement pour le marquage des « îlots » énonciatifs et des unités du dynamisme communicatif. »
\end{abstract}

Nous assistons, avec les compléments différés, à une mise en place progressive de l'apport informationnel, l'information étant plus pertinente lorsqu'elle est véhiculée par des éléments autonomes et rhématiques. Selon Halliday (1985), les principales différences entre productions orales et écrites proviennent essentiellement de la façon de présenter l'information. L' " information packaging », c'est-àdire les différents façonnages de l'information, appelleraient la mise en place de constructions ou formes grammaticales types : une forte concentration lexicale, la fragmentation phrastique en unités inférieures et distinctes, etc.

Le rythme que nous soumettent nos exemples étudiés renvoie au cheminement spontané de la réflexion du locuteur qui marque d'une nouvelle unité chaque nouvel élément informatif. Ces segments sont donnés comme informatifs, c'est-à-dire porteurs d'éléments pertinents pour la communication. Ils permettent de pointer un élément. L'ajout d'un nouvel élément dans le discours facilite bien souvent la mise en place d'une nouvelle unité syntaxique autonome. Le critère d'informativité intervient ici pour soutenir l'idée d'une nouvelle phrase. À chaque unité correspond un nouveau thème discursif. Au niveau textuel, ces segments jouent un rôle important : ils amènent progressivement l'information et assurent une certaine continuité. Le locuteur gagne en clarté d'une séquence à l'autre. Il pointe sur l'élément le plus informatif. C'est ce que nous avions en (6) :

(6) je travaillais pour une usine - Coder la même qu'il y a à Marseille /ou, X/à Saint-Marcel mais - à la Garenne Bezons à Paris - [CTFP : 27]

avec une progression du type, nous l'avons déjà mentionné :

$1 \rightarrow$ Je travaillais pour une usine.

$2 \rightarrow$ Cette usine est Coder.

$3 \rightarrow$ Cette usine Coder est la même qu'il y a à Marseille. 
Les fragments différés possèdent ainsi un degré élevé d'informativité, ce qui en suppose une rhématisation importante. Le caractère averbal appuie la focalisation sur un élément de la phrase qui précède ou sur un élément du contexte antérieur.

\section{Tests \& indices}

Les compléments différés ne peuvent constituer des noyaux à part entière que si et seulement si ils répondent à une série de tests.

\subsection{Le changement de modalité énonciative}

Lorsque l'ajout porte une valeur différente de ce qui précède, le caractère autonome est certain (Delorme \& Lefeuvre, 2004). L'autonomie énonciative des segments relevés peut donc être renforcée par un changement de modalité, différente de celle de la phase précédente :

(2a) ils ont essayé de casser le passage dans le quartier pour en faire un quartier tranquille [CFPP2000: Nicole Noroy $14^{\mathrm{e}}$ ]

(2b) Ils ont essayé de casser le passage dans le quartier. Pour en faire un quartier tranquille? (modalité interrogative)

(2c) ? ils ont essayé de casser le passage dans le quartier pas pour en faire un quartier tranquille (modalité négative)

(2d) Ils ont essayé de casser le passage dans le quartier. Pour en faire un quartier tranquille! (modalité exclamative d'affect)

Le changement de modalité s'effectue également sans aucun problème pour l'ensemble des caractérisations énonciatives :

(4a) ça m'avait frappée mais mais comme un coup de tonnerre [CTFP : 35]

(4b) ça m'avait frappée mais mais pas comme un coup de tonnerre (modalité négative)

(4c) Ça m'avait frappée. Mais comme un coup de tonnerre? (modalité interrogative)

(4d) Ça m'avait frappée. Mais comme un coup de tonnerre! (modalité exclamative d'affect)

\subsection{L'acceptation de marqueurs signalant un jugement de la part du locuteur}

Un second test consiste à observer si les compléments différés sans marqueurs acceptent différentes marques de la présence d'un jugement énonciatif du locuteur telles que les locutions "apparemment», «d'ailleurs », « après tout», «probablement», «peut-être», «sans doute», etc. Les marqueurs de prédication peuvent également renforcer le caractère prédicatif de l'énoncé. Ils sont « chargé[s] d'orienter l'élément averbal avec une valeur prédicative » (Delorme et Lefeuvre 2004 : 14). La prédication peut être signalée et renforcée par le marqueur négatif «pas», des adverbes d'intensité - «bien », « très », « plus », « trop », « tout »- ou des adverbes aspectuels - « encore », « enfin », « toujours », « souvent», etc.

(2a) ils ont essayé de casser le passage dans le quartier pour en faire un quartier tranquille [CFPР2000: Nicole Noroy $14^{\mathrm{e}}$ ]

(2e) Ils ont essayé de casser le passage dans le quartier. Surtout / apparemment / d'ailleurs, probablement / peut-être / sans doute pour en faire un quartier tranquille.

\subsection{La présence d'un terme modalisant}

Les différents termes introduisant les caractérisations énonciatives sont de sérieux indices d'autonomie énonciative : 
(4) ça m'avait frappée mais mais comme un coup de tonnerre [CTFP : 35]

Cela concerne les éléments « et », « donc », « déjà », « enfin », « d'abord », 《 toujours », « surtout », « d'ailleurs », « mais », etc. Ces différents introducteurs sont des indices d'autonomie de l'élément ajouté. Ils traduisent l'attitude adoptée par le locuteur à l'égard de son énoncé et devant sa propre énonciation : marques de jugement, termes d'adhésion, de doute, d'hypothèse, etc. Ces introducteurs signalent ainsi un changement de plan énonciatif dans le discours. La présence, par exemple, du relateur " et », tout comme celle de «mais », conduit à un décrochage énonciatif. Elle oblige à interpréter l'élément ajouté comme le signalement d'un changement de point de vue, comme le passage à un commentaire du locuteur, à une confirmation, à une précision, à une réorientation argumentative de l'énoncé, etc.

\subsection{Autonomie énonciative}

Les caractérisations modalisées telles que (8) :

(8a) donc moi j'y j'y j'y vais plusieurs fois mais en touriste [GRE03 : doreur]

peuvent fonctionner comme des énoncés autonomes, comme des réponses à une question :

(8b) Tu es allé plusieurs fois?

Oui, mais en touriste.

ou comme des énoncés en coénonciation :

(8c) L1 j'y vais plusieurs fois

L2 mais en touriste

Cette manipulation est également possible avec les compléments différés sans marqueur :

(9) L1: j'ai acheté des lunettes euh L2: oui qui sont très peu remboursées hein [Sabio, 2006 : 130]

\subsection{Test de la restriction}

Le test de la restriction peut également parfois conforter la possibilité pour les compléments différés de composer un noyau autonome pouvant être contrasté :

(8a) donc moi j'y j'y j'y vais plusieurs fois mais en touriste [GRE03 : doreur]

(8d) donc moi j'y j'y j'y vais plusieurs fois mais qu'en touriste

D'autres tests (clivage, acceptation de la négation) ou encore le recours à une glose verbale peuvent également nous orienter vers une prédication pleinement autonome :

(4a) ça m'avait frappée mais mais comme un coup de tonnerre [CTFP : 35]

(4e) ça m'avait frappée mais mais c'était comme un coup de tonnerre

(4f) ça m'avait frappée mais mais comme un coup de tonnerre ça m'avait frappée

(4g) ça m'avait frappée mais mais c'est comme un coup de tonnerre que ça m'avait frappée

\section{Proposition d'analyse à l'interface syntaxe - prosodie}

Nous avons exposé que les compléments différés peuvent être interprétés de trois façons différentes. Différents critères ou tests, que nous venons de décrire, peuvent orienter vers une de ces trois lectures. Cependant, en l'absence d'indices de segmentation suffisants, nous ne pouvons décider. La prosodie peut alors proposer des critères d'interprétation importants.

Nous avons vu dans notre premier point que le détachement est marqué par une rupture prosodique mettant en évidence deux périodes du type : 
(3) il me reste un morceau à mettre là // pour fermer euh le tour du cadre //[GRE03 : doreur]

Il convient ensuite de distinguer deux types de périodes s'opposant selon le contour intonatif qui précède. La structure prédicative de gauche peut en effet porter un intonème terminal (conclusif) ou non terminal (continuatif).

Nous pouvons ainsi envisager trois cas de figure possibles pour (2) et illustrées en prosodie par les découpages suivants :

(2a) ils ont essayé de casser le passage dans le quartier pour en faire un quartier tranquille [CFPP2000; Nicole Noroy $\left.14^{\mathrm{e}}\right]$

(2d) ils ont essayé de casser le passage dans le quartier pour en faire un quartier tranquille //

(2e) ils ont essayé de casser le passage dans le quartier $[\rightarrow] / /$ pour en faire un quartier tranquille //

(2f) ils ont essayé de casser le passage dans le quartier [ $[\downarrow] / /$ pour en faire un quartier tranquille //

En (2d), la structure conjointe est liée. Il n'y a qu'une seule période intonative. En (2e), la structure est disjointe, disloquée. Nous avons deux périodes. La première se termine par un intonème continuatif. En (2f), nous avons des structures autonomes. L'extrait est analysé en deux périodes, la première se conclut sur une intonation conclusive.

Ainsi, pour analyser les compléments différés, nous proposons, à partir de la prosodie, les critères suivants: (i) la présence ou non d'une rupture prosodique qui fera apparaître une ou deux périodes distinctes, (ii) le critère de terminalité ou de continuité prosodique précédant la première structure. Nous prendrons le critère de terminalité prosodique comme signe de clôture marquant la fin d'une séquence syntaxique. Le second critère découlera de l'actualisation du premier.

Pour les cas de périodes averbales, celles qui sont précédées d'un contour continuatif seront analysées comme des éléments détachés, suffixes, tandis que celles qui sont précédées d'un contour conclusif seront analysées comme des énoncés autonomes, syntaxiquement et énonciativement. Les segments averbaux ambigus non détachés prosodiquement seront intégrés au noyau de gauche.

Nous pouvons résumer cela par le tableau suivant :

\begin{tabular}{|l|l|l|l|}
\hline & Élément intégré & $\begin{array}{l}\text { Élément détaché } \\
\text { suffixe }\end{array}$ & $\begin{array}{l}\text { Élément autonome } \\
=\text { noyau (phrase) }\end{array}$ \\
\hline Rupture prosodique & - & + & + \\
\hline $\begin{array}{l}\text { Contour conclusif sur } \\
\text { l'élément qui précède }\end{array}$ & - & - & + \\
\hline
\end{tabular}

Figure 2 : Proposition d'analyse syntaxique des compléments différés à l'interface syntaxe - prosodie.

Les éléments énoncés sans rupture prosodique seront intégrés au noyau qui précède. C'est le cas de la relative en (10):

(10) ça a commencé par une paire de palmes et d'un tuba qui ont jamais vu la mer // et même jamais vu l'eau je crois bien // [CRFP : Plongée]

Les périodes averbales précédées d'un contour continuatif sur l'élément qui précède seront analysées comme des éléments détachés, suffixes. C'est le cas du groupe prépositionnel « sur un bateau un joli bateau » en (11):

(11) c'est un voyage en Égypte en Mer Rouge c'est une croisière // sur un un bateau un joli bateau // qui est arrêté maintenant // [CRFP : Plongée] 
Les périodes averbales précédées d'un contour conclusif sur l'élément qui précède seront analysées comme des noyaux, c'est-à-dire des éléments autonomes. C'est le cas de la relative en (11) :

(11) c'est un voyage en Égypte en Mer Rouge c'est une croisière // sur un un bateau un joli bateau // qui est arrêté maintenant // [CRFP : Plongée]

Cependant, les segments ajoutés du type de la relative en (11) ne sont pas totalement autonomes par rapport à la structure antérieure. L'ajout induit une construction $\varnothing$ qui correspond en réalité à la réactualisation de la phrase qui précède. Nous suivons ici en partie le point de vue de Culicover et Jackendoff (2005) qui préfèrent une analyse par regroupement de type paratactique entre une structure verbale et un «fragment» autonome syntaxiquement, tout de même relié à la construction précédente mais uniquement au niveau de l'interprétation.

Nous parlerons de réduplication implicite d'une structure verbale déjà émise. Ainsi pour (12) :

(12) c'est là que j'ai commencé à conduire - un camion - sans permis mais là - c'était l'exode on s'en foutait [CTFP : 27]

si l'énoncé suit le schéma prosodique :

c'est là que j'ai commencé à conduire $[\downarrow] / /$ un camion $[\downarrow] / /$ sans permis $[\downarrow] / /$

où $[\downarrow]$ indique un contour terminal, nous aurons trois unités distinctes à la fois prosodiquement, énonciativement et syntaxiquement. Nous ne pourrons donc parler, pour le groupe nominal « un camion » de complément direct du verbe "conduire » exprimé. Il spécifie en réalité ce même verbe "conduire » non répété (Avanzi, 2010 à par.). Ainsi :

$$
\begin{aligned}
& \text { c'est là que j'ai commencé à conduire } \\
& \text { [c'est là que j'ai commencé à conduire] un camion } \\
& \varnothing \rightarrow \quad \text { un camion } \\
& \varnothing \rightarrow \quad \text { sans permis }
\end{aligned}
$$

Ces ajouts correspondent en réalité à une focalisation averbale. Ils permettent de mettre l'accent sur certains éléments phrastiques, tout en amenant l'information progressivement, morcelant ainsi la structure modèle.

L'exemple (5) :

(5) d'ailleurs c'est à peu près le la configuration qu'ils ont gardée pour créer il y a quelques années ce fameux quartier vert // qui finalement a fini par tuer le quartier // parce qu'ils ont coupé totalement la la circulation // [CFPP2000 : Nicole Noroy $14^{\mathrm{e}}$ ]

qui contient deux périodes intonatives précédées toutes deux de contours continuatifs sera analysé en une seule phrase avec un noyau suivi de deux suffixes.

\section{Conclusion}

Nous avons ainsi, à travers cette étude, élaboré un modèle d'interprétation des compléments différés dans l’oral spontané, sujets à plusieurs interprétations : (i) élément intégré à la structure qui précède, (ii) élément détaché ou (iii) élément autonome.

Les exemples décrits ont permis d'illustrer les différentes variantes de découpages possibles pour un même texte. Pour traiter de ces réalisations, nous avons associé les notions de phrase et de période intonative. Au niveau prosodique, nous avons pris en compte deux critères : la présence ou non d'une rupture prosodique qui fait apparaître une ou plusieurs périodes intonatives distinctes et le critère de terminalité ou de continuité prosodique précédant la première structure. 
Ainsi, les éléments énoncés sans rupture prosodique sont intégrés au noyau qui précède. Les périodes précédées d'un contour mélodique continuatif sur la période qui précède seront analysées comme des suffixes. Les périodes précédées d'un contour mélodique conclusif sur la période antérieur seront analysées comme des éléments autonomes. Nous avons également introduit l'idée d'une construction $\varnothing$ correspondant à la réactualisation de la phrase précédente. Nous avons ainsi parlé de réduplication implicite d'une structure verbale déjà émise. La syntaxe est perçue sur ce point dans une dimension dynamique. Les phrases n'ont qu'une existence temporaire dans le flux de l'activité productive (pour l'énonciateur) et interprétation (pour le récepteur) (Le Goffic, 2008 : 330). Une phrase peut être achevée et reprise immédiatement pour y adjoindre un complément.

\section{Références bibliographiques}

Avanzi, M. (2005). Quelques hypothèses à propos de la structuration interne des périodes. Actes du symposium Interface Discours-Prosodie. Aix-en-Provence. 8-9 sept. 05. CD-ROM.

Avanzi, M. (2008). La différence entre micro- et macro-syntaxe est-elle marquée prosodiquement ? L'exemple des dispositifs clivés en il y a SN qui / Ø V. L'information Grammaticale. 119, 8-13.

Avanzi, M. (2010 à par.). Rattachement et fragmentation de la syntaxe par la prosodie. Travaux de linguistique « La Rection verbale, entre micro- et macro-syntaxe ». Numéro coordonné par Gautier, A., Pino Serrano, L. \& Van Raemdonck, D.

Avanzi, M. \& Lacheret-Dujour, A. (2009 à par.). Micro-syntaxe, macro-syntaxe : une prosodie toujours transparente? L'exemple des périodes asyndétiques en français parlé. In Beguelin, M-J., Avanzi, M. \& Corminboeuf, G. (éds). La parataxe. vol. 2 : Structures, marquages et exploitation discursive (Actes du colloque " La parataxe », Neuchâtel, 12-15 février 2007).

Avanzi, M., Lacheret-Dujour, A. \& Victorri, B. (2008). Analor, un outil d'aide pour la modélisation de l'interface prosodie - grammaire. Cahiers du CERLICO. 21, 27-46.

Bally, C. (1944). Linguistique générale et linguistique française. Berne : Francke.

Blanche-Benveniste, C. (1993). Une description linguistique du français parlé. Le Gré des langues. 5, 8-29.

Blanche-Benveniste, C. (1997). Approches de la langue parlée en français. Paris : Ophrys. coll. : « l'essentiel ».

Blanche-Benveniste, C (2002). Macro-syntaxe et micro-syntaxe : les dispositifs de la rection verbale. In Andersen, H. L. \& Nølke, H. (éds). Macro-syntaxe et macro-sémantique. Berne : Peter Lang, 95-118.

Blanche-Benveniste, C. (2003). Le recouvrement de la syntaxe et de la macro-syntaxe. In SCARANO, A. (éds). Macro-syntaxe et pragmatique : l'analyse linguistique de l'oral. Italie/Rome : Bulzoni, 53-75.

Blanche-Benveniste, C., Rouget, C. et Sabio, F. (2002). Choix de textes de français parlé, 36 extraits, Paris : Honoré Champion.

Blanche-Benveniste, C. et al. (1990). Le Français parlé : études grammaticales. Paris : Éditions du CNRS.

Bonhomme, M. (1998). Les figures clés du discours. Éditions du Seuil, Paris.

Branca-Rosoff, S., Fleury, S., Lefeuvre, F. \& Pires, M. (2009). Discours sur la ville. Corpus de Français Parlé Parisien des années 2000 (CFPP2000)http://ed268.univ-paris3.fr/CFPP2000/

Combettes, B. (2007). Les ajouts après le point : aspects syntaxiques et textuelles. In CHAROLLES M. et al. (éds). Parcours de la phrase. Mélanges offerts à Pierre Le Goffic. Paris : Ophrys, 119-132.

Culicover, P. W. \& Jackendoff, R. (2005). Simpler syntax. Oxford / New York : Oxford University Press.

Delic (2004). Présentation du Corpus de Référence du Français Parlé. RSFP 18, 11-42. [CRFP]

Delorme, B. \& Lefeuvre, F. (2004). De la prédication seconde à la prédication autonome. Verbum. XXVI. 3, 281-297.

Deulofeu, J. (2003). L'approche macro-syntaxique en syntaxe : un nouveau modèle de rasoir d'Occam contre les notions inutiles? SCOLIA. 16. Strasbourg : Publications de l'Université Marc Bloch, 77-95. 
Deulofeu, J. (2009). Pour une linguistique du rattachement. In Apotheloz D., Combettes B. \& Neveu F. (éds) Les linguistiques du détachement. Bern : Peter Lang, 229-250.

Halliday, M. A. K. (1985). Spoken and written language. Oxford : Oxford University Press.

Lacheret-Dujour, A. (2003). La prosodie des circonstants en français parlé. Louvain/Paris : Peeters.

Lacheret-Dujour, A. \& Victorri, B. (2002). La période intonative comme unité d'analyse pour l'étude du français parlé : modélisation prosodique et enjeux linguistiques. Verbum. 24/1-2, 55-73.

Lefeuvre, F. (1999). La phrase averbale en français. Paris : L'Harmattan.

Le Goffic, P. (1993). Grammaire de la Phrase Française. Paris : Hachette Supérieur.

Le Goffic, P. (2001). Pourquoi et comment une grammaire de la phrase. In Demarty-Warzee, J. \& Rousseau, J. Faire une grammaire, faire de la grammaire. Paris : Didier, 29-39.

Le Goffic, P. (2008). Phrase, séquence, période. In Van Raemdonck, D. (éd.) Modèles syntaxiques. La syntaxe à l'aube du XXI siècle. Bruxelles : Peter Lang, 329-356.

Sabio, F. (2006). Phrases et constructions verbales : quelques remarques sur les unités syntaxiques dans le français parlé. In Constructions verbales et production de sens. Actes du colloque organisé à Besançon les 26, 27 et 28 janvier 2006. Presses universitaires de Franche-Comté, 127-140.

Tanguy, N. (2009) Les segments averbaux, unités syntaxiques de l'oral. Thèse de doctorat. Université Sorbonne Nouvelle Paris 3.

${ }^{1}$ Les exemples indexés «CFPP2000 + référence du texte» sont issus du Corpus de Français Parlé Parisien, CFPP2000 (Branca et al., 2009), composé d'un ensemble d'interviews sur les quartiers de Paris et de la proche banlieue à partir d'un questionnaire portant sur la ville. Les exemples indexés « CTFP : numéro du texte » sont issus du corpus publié et accessible de Blanche-Benveniste et al. (2002) : Choix de Textes de Français parlé. Les exemples indexés «CRFP + référence du texte » sont issus du corpus de Référence du Français Parlé, constitué par l'équipe DELIC et rassemblant 134 enregistrements. Les exemples indexés « GRE03 + référence du texte sont issus du corpus GRE 03 constitué par l'université de Grenoble 3 et indexés dans la base CLAPI. 